\title{
Variational approximation of a constraint signal by a Mumford-Shah type energy functional
}

\author{
J. Rakotondralambo ${ }^{1}$, G. Michaille ${ }^{24}$, R. Brouzet ${ }^{24}$ and W. Puech ${ }^{34}$ \\ 1 Département de Mathématiques et informatique, Faculté des sciences \\ Université d'ANTANANARIVO, MADAGASCAR \\ e-mail: jrakoton@univ-antananarivo.mg \\ ${ }^{2}$ I3M Laboratory, UMR CNRS 5149 \\ University of MONTPELLIER II, FRANCE \\ e-mail: gerard.michaille@unimes.fr, robert.brouzet@unimes.fr \\ 3 LIRMM Laboratory, UMR 5506 CNRS \\ University of MONTPELLIER, FRANCE \\ e-mail: william.puech@lirmm.fr \\ ${ }^{4}$ University of NIMES, Place G. Péri, 30021 NIMES CEDEX 1, FRANCE
}

\begin{abstract}
In this paper, the main objective is to establish an existence result of a variational model in image segmentation constrained by a given vector field. In the one dimensional case, we give a discrete version converging in a variational way to the continuous model. We finally describe the numerical analysis of this model with application in image segmentation.
\end{abstract}

Keywords - Image processing theory, image segmentation.

\section{INTRODUCTION}

In this paper, we aim to describe a theoretical and numerical treatment of the following problem stemming from the theory of image segmentation:

$$
\begin{aligned}
& E(u)=\inf _{v \in S B V(\Omega)} E(v), \\
& E(v)=\int_{\Omega}|\nabla v|^{2} d x+\int_{S_{v}} f d \mathcal{H}^{N-1}+\int_{\Omega}|g-v|^{2} d x,
\end{aligned}
$$

where $\Omega$ is a domain in $\mathbf{R}^{N}$ and $g: \Omega \rightarrow \mathbf{R}$

The density $f$ is the extended real-valued function $v \mapsto$ $1+I_{A}(v)$ where $A$ is the set of all the functions $v$ in $S B V(\Omega)$ satisfying, for a given $a \in \mathbf{R}$, the condition:

$$
[v](x) \nu_{v}(x) . \Phi(x) \geq a,
$$

for $\mathcal{H}^{N-1}\left\lfloor S_{v}\right.$ a.e. $x$ in $\Omega$. We point out that the energy $E$ is the perturbation of the so called Mumford-Shah energy [6] $f \equiv 1)$ by the indicator function $I_{A}$ of the set $A$ (i.e $I_{A}(v)=0$ if $v \in$ $A,+\infty$ otherwise). The vector valued function $\Phi$ belonging to $\mathcal{C}\left(\bar{\Omega}, \mathbf{R}^{N}\right)$, may be considered, when $N=2$, as a constraint on the outline of an image $g$ in computer vision: the outlines of the image having a jump of grey level in the direction of $\Phi$ more than $a$ are selected by the model. In the one dimensional case it leads to detect some selected discontinuities of the signal $g$.

In the paper $\mathcal{L}$ denotes the Lebesgue measure restricted to $\Omega$, $S B V(\Omega)$ the space of all the functions $v$ of bounded variation whose distributional gradient is of the form $D v=\nabla v \mathcal{L}+$ $[v] \nu_{v} \mathcal{H}^{N-1}\left\lfloor S_{v}\right.$. We denote by $[v]:=u^{+}-u^{-}$the jump of $v$ through the jump set $S_{v}, \nu_{v}$ is the unit normal to $S_{v}$ and $\mathcal{H}^{N-1}\left\lfloor S_{v}\right.$ is the restriction to $S_{v}$ of the $N-1$-dimensional
Hausdorff measure. In the one dimensional case, we adopt the following notation for the structure of the distributional derivative of any $v$ in $S B V(0,1): v^{\prime}=\dot{v} d t+[v] \mathcal{H}^{0}\left\lfloor S_{v}\right.$. In this specific case, $[v](t)$ is nothing but the classical jump of $v$ at $t \in S_{v}$ and $\mathcal{H}^{0}$ is the counting measure so that $\int[v] \mathcal{H}^{0}\left\lfloor S_{v}=\right.$ $\sum_{t \in S_{v}}[v] \delta_{t}$. For more about $S B V$ spaces, we refer the reader to [4].

In Section 2 we establish the existence of a solution $u$ of the problem. In Section 3 we define a variational discrete model in the case $N=1$. Let $E_{h}$ be some suitable functional expected to describe the discrete energy associated with the above functional $E, h$ denoting the step of discretization. We say that the problem inf $E_{h}$ is a variational discrete model of inf $E$ if $\min E_{h}$ converges to $\min E$ when $h$ goes to zero and if every minimizer $u_{h}$ of $E_{h}$ tends in $L^{1}(0,1)$ to a minimizer of $E$. Provided that $\left(u_{h}\right)_{h>0}$ be compact, an appropriate convergence for the sequence of functionals $\left(E_{h}\right)_{h>0}$ leading to this objective, is the so called $\Gamma$-convergence of the sequence $\left(E_{h}\right)_{h>0}$ to the functional $E$. Section 4 is devoted to the description of an algorithm giving a solution $u_{h}$ of the discrete problem $\min E_{h}$. We conclude the paper by giving some numerical experiments with application in image segmentation. Previous work have all ready applied variational models to image segmentation [8], [9], [10], [2].

\section{EXISTENCE OF A SOLUTION}

We equip $S B V(\Omega)$ with the norm $\|\cdot\|_{S B V(\Omega)}$ defined by:

$$
\|v\|_{B V(\Omega)}=\int_{\Omega}|v| d x+\int_{\Omega}|D v|
$$

where $\int_{\Omega}|D v|$ is the total mass of the total variation $|D v|$ of the measure $D v$.

We say that a sequence $\left(u_{n}\right)_{n \in \mathbf{N}}$ weakly converges to $u$ in $B V(\Omega)$ iff $u_{n} \rightarrow u$ strongly in $L^{1}(\Omega)$ and $D u_{n} \rightarrow D u$ weakly in the sense of measures in $\mathcal{M}\left(\Omega, \mathbf{R}^{N}\right)$.

Let $\left(u_{n}\right)_{n \in \mathbf{N}}$ be a minimizing sequence of inf $E(v)$. 
From Ambrosio's compactness theorem (see [1], [4]), one can establish the weak lower-semi-continuity of the functional $E$ together with existence of a weak cluster point $\bar{u}$ of $\left(u_{n}\right)_{n \in \mathbf{N}}$. Combining these two arguments gives $E(\bar{u})=$ $\inf E(v)$. We have established

Theorem 1: There exists at least one minimizer of the problem

$$
\inf _{v \in A}\left\{\int_{\Omega .}|\nabla v|^{2} d x+\mathcal{H}^{N-1}\left(S_{v}\right)+\int_{\Omega}|g-v|^{2} d x\right\}
$$

The following compactnes theorem is due to L. Ambrosio (see [1], [4]).

Theorem 2: Let $\left(u_{n}\right)_{n}$ be a sequence of elements in $S B V(\Omega)$ satisfying

(i) $\sup _{n \in \mathbf{N}}\left\{\left\|u_{n}\right\|_{B V(\Omega)}\right\}<+\infty$,

(ii) the sequence $\left(\nabla u_{n}\right)_{n \in \mathbf{N}}$ is equi-integrable,

(iii) the sequence $\left(\mathcal{H}^{N-1}\left(S_{u_{n}}\right)\right)_{n \in \mathbf{N}}$ is uniformly bounded.

Then one can extract a subsequence $\left(u_{n_{k}}\right)_{k \in \mathbf{N}}$ which converges to some $u \in S B V(\Omega)$ such that

$$
\begin{aligned}
& u_{n_{k}} \rightarrow u \text { in } L_{l o c}^{1}(\Omega), \\
& \nabla u_{n_{k}} \rightarrow \nabla u \text { weakly in } L^{1}\left(\Omega, \mathbf{R}^{N}\right), \\
& J u_{n_{k}} \rightarrow J u \text { weakly in } \mathcal{M}\left(\Omega, \mathbf{R}^{N}\right), \\
& \mathcal{H}^{N-1}\left(S_{u}\right) \leq \liminf _{k \rightarrow+\infty} \mathcal{H}^{N-1}\left(S_{u_{n_{k}}}\right) .
\end{aligned}
$$

Let $\left(u_{n}\right)_{n \in \mathbf{N}}$ be a minimizing sequence of inf $E(v)$. From classical argument stemming from measure theory, one can establish the weak lower-semicontinuity of the functional $E$. On the other hand, applying Theorem 2, one can easily see that there exists a weak cluster point $\bar{u}$ of $\left(u_{n}\right)_{n \in \mathbf{N}}$. Combining these two arguments gives $E(\bar{u})=\inf E(v)$. We have established

Theorem 3: There exists at least one minimizer of the problem

$$
\inf _{v \in A}\left\{\int_{\Omega}|\nabla v|^{2} d x+\mathcal{H}^{N-1}\left(S_{v}\right)+\int_{\Omega}|g-v|^{2} d x\right\}
$$

\section{DisCRETE MODEL IN ONE DIMENSIONAL CASE}

We assume here that $\Phi>0$ on $[0,1]$ and $a>0$. For $h=\frac{1}{N}$ we consider the following density defined for every $(t, e) \in$ $(0,1) \times \mathbf{R}$ by:

$$
W_{h}(t, e):=\left\{\begin{array}{l}
e^{2} \text { if } e \leq e_{h}(t) \\
\frac{1}{h} \text { if } e>e_{h}(t)
\end{array}\right.
$$

where $e_{h}(t)=\frac{a}{h \Phi(t)}$ and set $\tilde{W}_{h}(t, e)=W_{h}(i h, e)$ if $t \in$ $[i h,(i+1) h[$.

Let $\mathcal{A}_{h}(0,1)$ denote the set of all the continuous functions in $(0,1)$, affine on each interval $(i h,(i+1) h)$ of $(0,1)$ and bounded by $\|g\|_{\infty}$. We define the functional:

$$
F_{h}(v):=\left\{\begin{array}{l}
\int_{0}^{1} \tilde{W}_{h}\left(t, v^{\prime}(t)\right) d t \text { if } v \in \mathcal{A}_{h}(0,1), \\
+\infty \text { if } v \in L^{1}(0,1) \backslash \mathcal{A}_{h}(0,1) .
\end{array}\right.
$$

Our objective is to establish the $\Gamma$-convergence of the functional $v \mapsto E_{h}(v):=F_{h}(v)+\int_{0}^{1}|g(i h)-v|^{2} d x$ to the energy functional $E$ when $L^{1}(0,1)$ is equipped with its strong topology. In order to make relevant our variational approximating scheme, we must establish the following compactness result whose proof is a straightforward consequence of the behavior of $W_{h}$ and of the compactness of the embedding $W^{1,1}(0,1) \subset L^{1}(0,1)$.

Proposition 1: Let $\left(u_{h}\right)_{h>0}$ be a sequence of $L^{1}(0,1)$ satisfying

$$
E_{h}\left(u_{h}\right)=\inf \left\{E_{h}(v): v \in L^{1}(0,1)\right\}
$$

Then, there exist a subsequence (not relabeled) and $u \in$ $L^{1}(0,1)$ such that $u_{h} \rightarrow u$ strongly in $L^{1}(0,1)$.

Existence of $u_{h}$ above is obtained by arguments similar to those of the proof of Theorem 3. We now establish the $\Gamma$ convergence of $E_{h}$ to $E$, a notion of convergence introduced by De Giorgi and Franzoni. For overview, we refer the reader to [3] and references therein.

Theorem 4: Let $u \in L^{1}(0,1)$. Then the sequence $\left.E_{h}\right)_{h>0}$ $\Gamma$-converges to $E$, i.e., the two following statements hold:

$\left(\Gamma_{1}\right)$ there exists a sequence $\left(u_{h}\right)_{h>0}$ strongly converging to $u$ in $L^{1}(0,1)$ such that:

$$
\limsup _{h \rightarrow 0} E_{h}\left(u_{h}\right) \leq E(u)
$$

$\left(\Gamma_{2}\right)$ for every $u \in L^{1}(0,1)$ and every sequence $\left(u_{h}\right)_{h>0}$ strongly converging to $u$, we have:

$$
E(u) \leq \liminf _{h \rightarrow 0} E_{h}\left(u_{h}\right) .
$$

We should point out that this convergence is variational, i.e., every cluster point of the sequence $\left(u_{h}\right)_{h>0}$ given in Proposition 1 is a minimizer of $E$, and $\min \left\{E_{h}(v): v \in\right.$ $\left.L^{1}(0,1)\right\}$ converges to $\min \left\{E(v): v \in L^{1}(0,1)\right\}$. For a proof of $\left(\Gamma_{1}\right)$ and $\left(\Gamma_{2}\right)$, we refer the reader to [5].

\section{DESCRIPTION OF AN ALGORITHM FOR THE DISCRETE MODEL}

In this section, we would like to describe an algorithm for the computation of a solution of the discrete variational model stated in the previous section :

$$
E_{h}(u)=\min _{v \in \mathcal{A}_{h}(0,1)}\left(F_{h}(v)+\int_{0}^{1}|g(i h)-v|^{2} d x\right) .
$$

Obviously, one can rewrite the energy $E_{h}$ as a functional defined in $\mathbf{R}^{N+1}$ and the discrete problem becomes:

$$
\begin{aligned}
E_{h}(u) & =\min _{v \in \mathbf{R}^{N+1}}\left(\sum_{\left\{i: v_{i+1}-v_{i} \leq \frac{a}{\Phi(i h)}\right\}} \frac{\left|v_{i+1}-v_{i}\right|^{2}}{h}\right. \\
& \left.+\mathcal{H}^{0}\left(\left\{i: v_{i+1}-v_{i}>\frac{a}{\Phi(i h)}\right\}\right)\right)
\end{aligned}
$$

where $v=\left(v_{0}, \ldots, v_{N}\right)$.

The strategy consists now in conditioning the minimization by fixing:

a) firstly the cardinal of the set $\left(\left\{i: v_{i+1}-v_{i}>\frac{a}{\Phi(i h)}\right\}\right.$ that we will call fracture set, 
b) secondly, for each fixed cardinal, the site of such fracture set. The problem may be written : find $u$ in $\mathbf{R}^{N+1}$ solution of:

$$
\begin{aligned}
& \min _{k=0, \ldots, N-1} \min _{F_{k}} \min _{v \in \mathbf{R}^{N-k}, v_{i+1}-v_{i} \leq \frac{\alpha}{\Phi(i h)}} \\
& \left(\sum_{i \notin F_{k}} \frac{\left|v_{i+1}-v_{i}\right|^{2}}{h}+k+\sum_{i \notin F_{k}} h\left|g(i h)-v_{i}\right|^{2}\right) .
\end{aligned}
$$

Note that we have replaced the sum $\sum_{i=0}^{N} h\left|g(i h)-v_{i}\right|^{2}$ by the sum $\sum_{i \notin F_{k}} h\left|g(i h)-v_{i}\right|^{2}$ in the definition of the discrete energy. The first minimization problem:

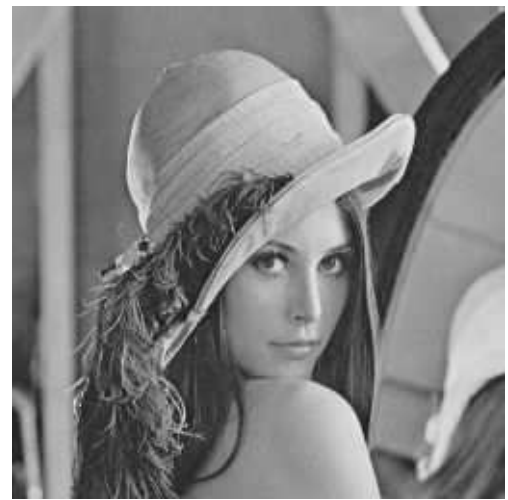

$\min _{v \in \mathbf{R}^{N-k}, v_{i+1}-v_{i} \leq \frac{\alpha}{\Phi(i h)}}\left(\sum_{i \notin F_{k}} \frac{\left|v_{i+1}-v_{i}\right|^{2}}{h}+k+\sum_{i \notin F_{k}} h\left|g(i h)-v_{i}\right|^{2}\right)$

Fig. 1. Original image of Lena.

consists in solving the classical quadratic optimization problem

$$
\min _{\mathbf{R}^{N-k}}\left(\sum_{i \notin F_{k}} \frac{\left|v_{i+1}-v_{i}\right|^{2}}{h}+\sum_{i \notin F_{k}} h\left|g(i h)-v_{i}\right|^{2}\right),
$$

and to take into account the solution $u_{k, F_{k}}$ if and only if its components satisfy the constraint $v_{i+1}-v_{i} \leq \frac{\alpha}{\Phi(i h)}$.

It is easily seen that (2) is equivalent to the linear problem:

$$
\left(\mathcal{A}^{T} \mathcal{A}+h^{2} I\right) v=h^{2} \tilde{g}_{h}
$$

where $I$ is the $(N-k) \times(N-k)$ identity matrix and $\mathcal{A}$ is a $(N-k) \times(N-k)$ matrix whose entries are -1 or 1 on the diagonal $i=j$ and 1 or 0 on the diagonal $j=i+1$.

\section{NUMERICAL EXPERIMENTS}

The problem is equivalent to the linear problem:

$$
\left(\begin{array}{cccc}
b_{0} & a_{0} & \cdots & 0 \\
a_{0} & b_{1} & \cdots & 0 \\
\vdots & \ddots & \ddots & \vdots \\
0 & \cdots & b_{n-1} & a_{n-1}
\end{array}\right)\left(\begin{array}{c}
v_{0} \\
v_{1} \\
\vdots \\
v_{n-1}
\end{array}\right)=\left(\begin{array}{c}
g_{0} \\
g_{1} \\
\vdots \\
g_{n-1}
\end{array}\right)
$$

where

$$
\left\{\begin{array}{lll}
b_{0}=b_{n-1} & =1+\frac{1}{h^{2}} \\
b_{i} & =1+\frac{2}{h^{2}} & \text { for } 1 \leq i \leq n-1 \\
a_{i} & =-\frac{1}{h^{2}} & \text { for all } \mathrm{i}
\end{array}\right.
$$

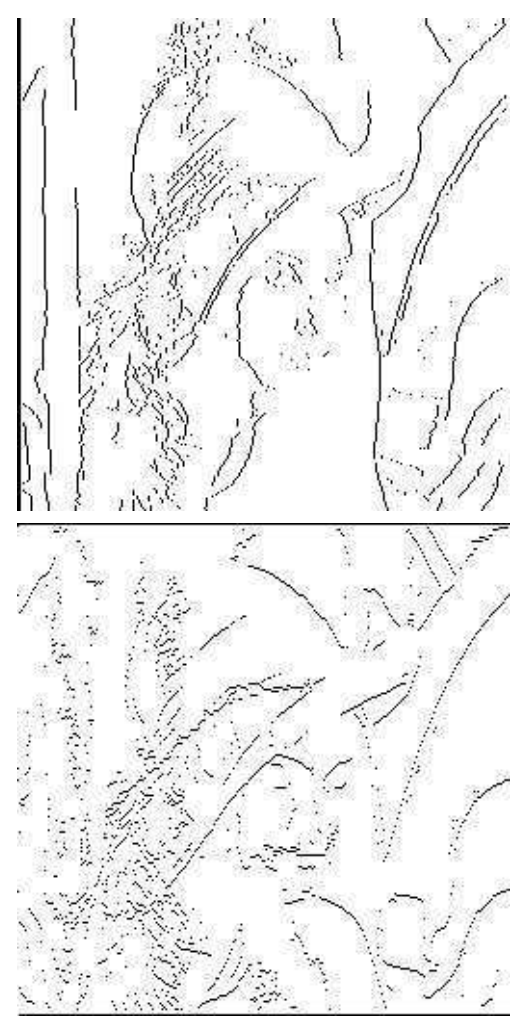

Fig. 2. Without filter, a) On line, b) On column.

To generalize, we introduce coefficients $\alpha, \beta$ and $\gamma$ in $E(v)$ and put:

$$
E(v)=\alpha \int_{\Omega}|\nabla v|^{2} d x+\beta \int_{\Omega}|g-v|^{2} d x+\gamma \int_{S_{v}} f d \mathcal{H}^{N-1} .
$$

We now give some reconstructed pictures obtained with various values of coefficients $\alpha, \beta$ and $\gamma$ and various values of the number $\frac{a}{\Phi(i h)}$ that we call filter. For working in one dimension, we scan the picture on line and on column in order to superpose the both. We limit the computation by taking $\#\left(F_{k}\right)=1$.

Without filter, from the original image of Lena, Fig.1, we obtain the detection on line, Fig.2.a, and on column, Fig.2.b. 
By superposition of the results of the Fig.2.a and b we have a full detection illustrated Fig.3. For the on line detection, Fig.4 and 5 , we have applied several values for the filter and for $\alpha, \beta$ and $\gamma$. In particular, for the Fig. 4.c, 4.d and 5, we have separated the pixels in two parts. The values of the parameters $\alpha, \beta$ and $\gamma$ have been fixed experimentally.

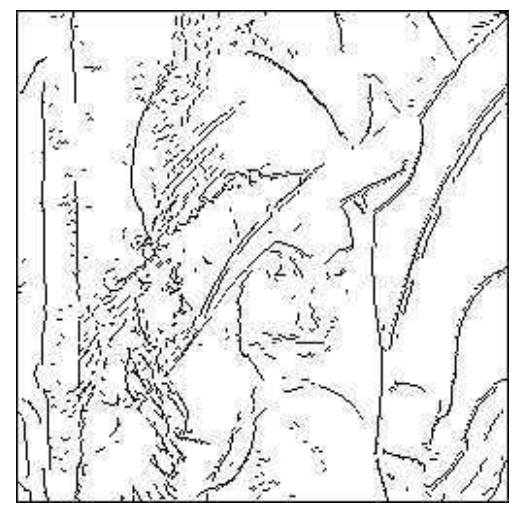

Fig. 3. By superposition of Fig.2.a and 2.b

For the on column detection, Fig.6, we have also applied several values for the filter and for $\alpha, \beta$ and $\gamma$. Finally, we illustrate Fig. 7 the superposition of the on line and on column detections.

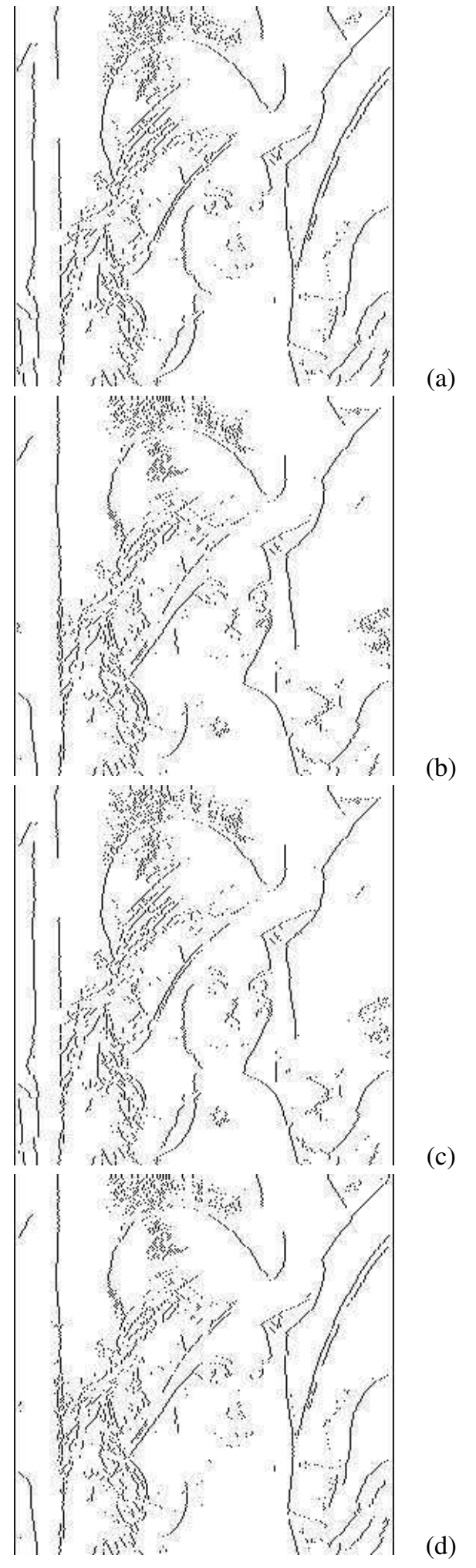

Fig. 4. On line, a) filter $=30$ and $\alpha=\beta=\gamma=1$, b) filter $=0.001$, $\alpha=200, \beta=0.01$ and $\gamma=0.1$. c) With two filters: filter1 $=0.001$ for pixels between 0 and 128 with $\alpha=200, \beta=0.01$ and $\gamma=0.1$, filter $2=30$ for pixels between 129 and 255 with $\alpha=\beta=\gamma=1$, d) With two filters: filter $1=30$ for pixels between 0 and 128 with $\alpha=\beta=\gamma=1$, filter2 = 0.001 for pixels between 129 and 255 with $\alpha=200, \beta=0.01$ and $\gamma=0.1$. 


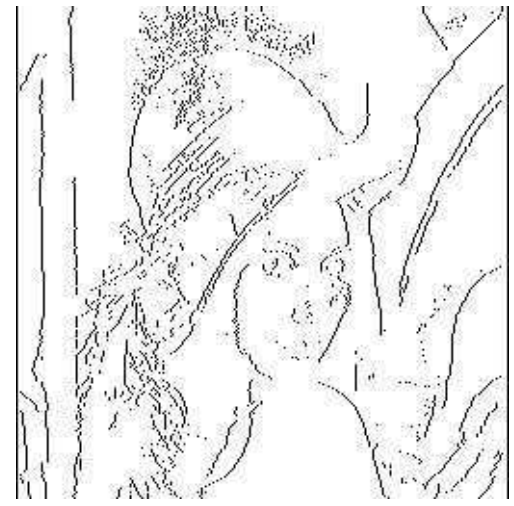

Fig. 5. On line with two filters: filter $1=0.001$ for pixels between 154 and 196 with $\alpha=200, \beta=0.01$ and $\gamma=0.1$, filter $2=30$ for pixels between 0 and 153, and from 197 until 255, with $\alpha=\beta=\gamma=1$.

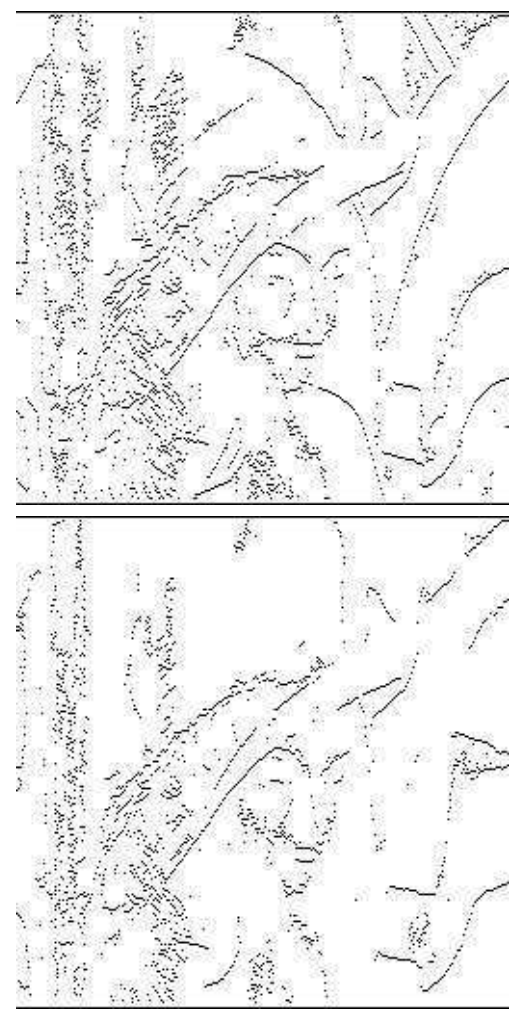

(a)

Fig. 6. On column, a) filter $=30, \alpha=\beta=\gamma=1$, b) filter $=0.001$, $\alpha=200, \beta=0.01$ and $\gamma=0.1$.

\section{CONCLUSION}

In this paper we have generalized the well known one dimensional discretization of the Mumford-Shah functional by taking into account a constraint on the outline (i.e. the filter). We have illustrated the method by scanning a picture on line and on column in order to superpose the both. Actually, the used filters in line and column could be considered as the projections of a given two dimensional vector field on the picture. In perspective of this work, we are planning to compare our method with level set methods [7].

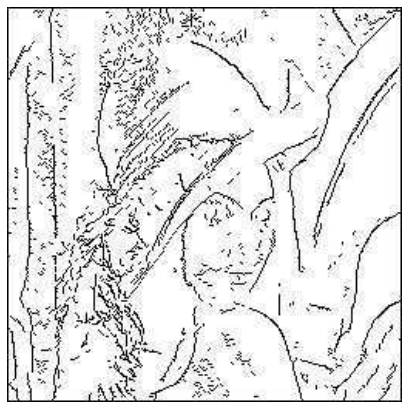

(a)

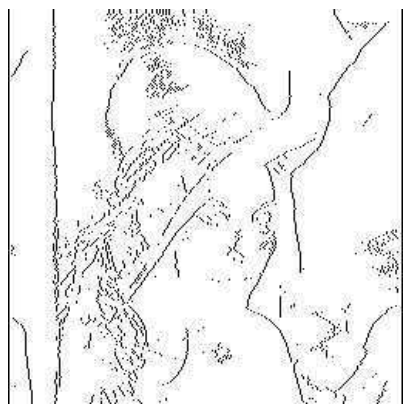

(b)
Fig. 7. a) Superposition of Fig.5 and Fig.6.a, b) Superposition of Fig.5 and Fig.6.b.

\section{REFERENCES}

[1] L. Ambrosio. A Compactness Theorem for a Special Class of Functions of Bounded Variation. Boll. Un. Mat. Ital. 3-B, 857-881, 1989.

[2] L. Ambrosio, L. Faina and R. March. Variational Approximation of a Second Order Free Discontinuity Problem Computer Vision, SIAM J. Math. Anal., 32, 1171

[3] H. Attouch. Variational Convergence for Functions and Operators Applicable Mathematics Series. Pitman Advanced Publishing Program, 1985.

[4] A. Braides. Approximation of Free-Discontinuity Problems. Lecture Notes in Mathematics, 1694, Springer Verlag, Berlin, 1998.

[5] R. Brouzet, G. Michaille, W. Puech and J. Rakotondralambo. Detection and Analysis of Discontinuities of a Constrained Signal. Prepublication of the Department of mathematics of the University of Montpellier 2, 2006.

[6] E. De Giorgi, M. Carriero and A. Leaci. Existence Theorem for a Minimum Problem with Free Discontinuity Set. Arch. Rational Mech. Anal., 108, 195-218, 1989.

[7] K. Djemal, W. Puech and B. Rossetto. Automatic Active Contours Propagation in a Sequence of Medical Images. International Journal of Images and Graphics. 6(2):267-292, 2006.

[8] J.M. Morel and S. Solimini. Density Estimates for the Boundaries of Optimal Segmentations. CRASS I-Mathmatique 312(6): 429-432, 1991.

[9] D. Mumford and J. Shah. Optimal Approximation by Piecewise Smooth Functions and Associated Variational Problems. Comm. Pure Appl. Math. 42:577-685, 1989.

[10] Y. Wang. Existence and Regularity of Solutions to a Variational Problem of Mumford and Shah: A Constructive Approach. SIAM J. Optim. 5, 892 1995. 